\title{
Global Attractors of the Extensible Plate Equations with Nonlinear Damping and Memory
}

\author{
Xiaobin Yao and Qiaozhen Ma \\ College of Mathematics and Statistics, Northwest Normal University, Lanzhou 730070, China \\ Correspondence should be addressed to Qiaozhen Ma; maqzh@nwnu.edu.cn
}

Received 12 January 2017; Accepted 2 March 2017; Published 4 April 2017

Academic Editor: Hugo Leiva

Copyright (C) 2017 Xiaobin Yao and Qiaozhen Ma. This is an open access article distributed under the Creative Commons Attribution License, which permits unrestricted use, distribution, and reproduction in any medium, provided the original work is properly cited.

We prove in this paper the existence of a global attractor for the plate equations of Kirchhoff type with nonlinear damping and memory using the contraction function method.

\section{Introduction}

Let us consider the long-time behavior for the following Kirchhoff plate equations with fading memory and nonlinear damping:

$$
\begin{aligned}
& u_{t t}+g\left(u_{t}\right)+\alpha \Delta^{2} u-\int_{0}^{\infty} \mu(s) \Delta^{2} u(t-s) d s+\lambda u \\
& \quad+\left(p-\beta \int_{\Omega}|\nabla u|^{2} d x\right) \Delta u+f(u)=h(x), \\
& u=\frac{\partial u}{\partial \nu}=0 \quad \text { on } \partial \Omega \times \mathbb{R}, \\
& u(x, \tau)=u_{0}(x, \tau), \\
& u_{t}(x, \tau)=\partial_{t} u_{0}(x, \tau),
\end{aligned}
$$

$$
(x, \tau) \in \Omega \times(-\infty, 0],
$$

where $\Omega \subset \mathbb{R}^{N}$ is a bounded domain with smooth boundary $\partial \Omega ; \alpha, \lambda$, and $\beta$ are positive constants, $p \in \mathbb{R}, h \in L^{2}(\Omega) ; \nu$ is the unit outer normal on $\partial \Omega ; u_{0}: \Omega \times(-\infty, 0] \rightarrow \mathbb{R}$ is the prescribed past history of $u$.

Problem (1) arises from the isothermal viscoelastic theory; it describes a fourth-order viscoelastic plate with a lower order perturbation and also models transversal vibrations of a thin extensible elastic plate in a history space, which is established based on the framework of elastic vibration by Woinowsky-Krieger [1] and Berger [2], and can be seen as an elastoplastic flow equation with some kind of memory effects (see [3,4] for details). The convolution term means that the stress at any instant $t$ depends on the whole history of strains which the material has undergone and produced a weak damping mechanism (see $[5,6]$ ).

In the case where $\mu(s) \equiv 0, p=\beta=0$, (1) becomes the normal plate equation which has been treated in many papers such as [7-14]. For instance, the authors investigated the existence of the compact attractor for the plate equation on both the bounded domain $[8,10,13]$ and the unbounded domain in $[7,11,12]$, respectively. Yue and Zhong [9] proved the existence of global attractors to the plate equations when the nonlinear function satisfies the critical exponent in a locally uniform space. In [14], the authors studied the existence of the random attractor for the stochastic strongly damping plate equations with additive noise and critical nonlinearity.

The case of $p=\beta \equiv 0$ in problem (1) has been studied by several authors (see $[2,5,15-23]$ and references therein). For instance, Wu [15] scrutinized the existence of global attractors for the nonlinear plate equation with thermal memory effects due to non-Fourier heat flux laws when $g\left(u_{t}\right)=-\Delta u_{t}$. Recently, Conti and Geredeli [5] paid attention to the existence of a smooth global attractor for the nonlinear viscoelastic equations with memory, in which they required the nonlinear damping $g\left(u_{t}\right)$ to be the polynomial growth. Shen and Ma [21] studied the existence of the random attractor for plate equations with memory and additive 
white noise. On the other hand, the asymptotic behavior of solutions for the extensible plate equations without memory affection was studied by several authors in [24-27].

We focus on the existence of the extensible plate equations with nonlinear damping and history memory in the present paper. To prove the existence of a compact global attractor, the key goal is to establish the compact property of the semigroup associated with the dynamical system. Regarding problem (1), we need to overcome the following difficulties. One difficulty is caused by the critical nonlinearity and nonlinear damping. In order to overcome these obstacles, we apply the contraction function method into our problem. Another difficulty is brought about by the memory kernel, because there is no compact embedding in the history space; besides, we cannot use the finite rank method. We solve this term by introducing a new variable and defining an extending phase space (see [20] for details). In addition, the terms ( $p-$ $\left.\beta \int_{\Omega}|\nabla u|^{2} d x\right) \Delta u(p \in \mathbb{R})$ make the estimates more complex, so we have to deal with them through accurate computation. Our main result is Theorem 12 .

As in [18], we define

$$
\eta=\eta^{t}(x, s)=u(x, t)-u(x, t-s),
$$

$$
(x, s) \in \Omega \times \mathbb{R}^{+}, t \geq 0 .
$$

By assuming that $\mu \in L^{1}\left(\mathbb{R}^{+}\right)$and taking $\alpha=1+$ $\int_{\infty}^{0} \mu(s) d s$, the original memory term can be rewritten as

$$
\begin{aligned}
& \int_{0}^{\infty} \mu(s) \Delta^{2} u(t-s) d s \\
& \quad=\left(\int_{0}^{\infty} \mu(s) d s\right) \Delta^{2} u-\int_{0}^{\infty} \mu(s) \Delta^{2} \eta^{t}(s) d s,
\end{aligned}
$$

and then problem (1) can be transformed into the following system:

$$
\begin{array}{r}
u_{t t}+g\left(u_{t}\right)+\Delta^{2} u+\int_{0}^{\infty} \mu(s) \Delta^{2} \eta^{t}(s) d s+\lambda u \\
+\left(p-\beta \int_{\Omega}|\nabla u|^{2} d x\right) \Delta u+f(u)=h(x) \\
\text { in } \Omega \times \mathbb{R}^{+},
\end{array}
$$

$$
\eta_{t}=-\eta_{s}+u_{t}, \quad \text { in } \Omega \times \mathbb{R}^{+} \times \mathbb{R}^{+} .
$$

Here, (5) is obtained by differentiating (2). Initial-boundary value conditions are given as follows:

$$
\begin{aligned}
u & =\frac{\partial u}{\partial v}=0 \quad \text { on } \partial \Omega \times \mathbb{R}^{+}, \\
\eta & =\frac{\partial \eta}{\partial v}=0, \quad \text { on } \partial \Omega \times \mathbb{R}^{+} \times \mathbb{R}^{+}, \\
u(x, 0) & =u_{0}(x), \\
u_{t}(x, 0) & =u_{1}(x), \\
\eta^{t}(x, 0) & =0, \\
\eta^{0}(x, s) & =\eta_{0}(x, s),
\end{aligned}
$$

where

$$
\begin{aligned}
u_{0}(x) & =u_{0}(x, 0), \quad x \in \Omega, \\
u_{1}(x) & =\left.\partial_{t} u_{0}(x, t)\right|_{t=0}, \quad x \in \Omega, \\
\eta_{0}(x, s) & =u_{0}(x, 0)-u_{0}(x,-s), \quad(x, s) \in \Omega \times \mathbb{R}^{+} .
\end{aligned}
$$

This paper is organized as follows: in Sections 2 and 3, we make some preparations for our consideration; in Section 4, we will show the existence of bounded absorbing set and compact global attractors.

\section{Assumptions}

The following conditions are necessary for our main result.

Concerning the nonlinear term $f \in C^{1}(\mathbb{R})$, there exists a constant $k_{0}>0$ such that

$$
\left|f^{\prime}(s)\right| \leq k_{0}\left(1+|s|^{\rho}\right),
$$

where

$$
\begin{aligned}
& 0<\rho \leq \frac{4}{N-4} \text { if } N \geq 5, \\
& \rho>0 \quad \text { if } 1 \leq N \leq 4 .
\end{aligned}
$$

Condition (9) implies that $H_{0}^{2}(\Omega) \hookrightarrow L^{2(\rho+1)}(\Omega)$. Also, we say that $\rho=4 /(N-4)$ is a critical exponent for the growth of $f(u)$ when $N \geq 5$. In addition, we assume that

$$
\liminf _{|s| \rightarrow \infty} \frac{f(s)}{s}>-\lambda_{1}
$$

where $\lambda_{1}>0$ is the principal eigenvalue of $\Delta^{2}$ in $H_{0}^{2}(\Omega)$.

The nonlinear damping function $g \in C^{1}(\mathbb{R})$ satisfies

$$
\begin{aligned}
g(0) & =0, \\
g^{\prime}(s) & \geq l>0, \\
|g(s)| & \leq k_{1}\left(1+|s|^{q}\right),
\end{aligned}
$$

with $1 \leq q<\infty$ if $N \leq 4$ and $1 \leq q \leq(N+4) /(N-4)$ if $N \geq 5$.

With respect to the memory kernel $\mu$, we assume that

$$
\begin{gathered}
\int_{0}^{\infty} \mu(s) d s=\mu_{0}>0, \quad \mu \in C^{1}\left(\mathbb{R}^{+}\right) \cap L^{1}\left(\mathbb{R}^{+}\right), \\
\mu^{\prime}(s) \leq 0 \leq \mu(s), \quad \forall s \in \mathbb{R}^{+},
\end{gathered}
$$

and that there exists a constant $k_{2}>0$ such that

$$
\mu^{\prime}(s)+k_{2} \mu(s) \leq 0, \quad \forall s \in \mathbb{R}^{+} .
$$

Now, we consider the Hilbert spaces that will be used in our analysis. Let

$$
\begin{aligned}
& H=V_{0}=L^{2}(\Omega), \\
& V=V_{1}=H_{0}^{2}(\Omega),
\end{aligned}
$$


equipped with the respective inner products and norms,

$$
\begin{gathered}
(u, v)_{V}=(\Delta u, \Delta v), \\
\|u\|_{V}=\|\Delta u\|_{2},
\end{gathered}
$$

where $(\cdot, \cdot)$ is $L^{2}$-inner product and we use $\|\cdot\|_{p}$ to denote $L^{p}$-norms.

We define the following weighted $L^{2}$-space:

$$
\begin{aligned}
& L_{\mu}^{2}\left(\mathbb{R}^{+} ; V\right) \\
& \quad=\left\{\eta: \mathbb{R}^{+} \longrightarrow V \mid \int_{0}^{\infty} \mu(s)\|\eta(s)\|_{V}^{2} d s<\infty\right\},
\end{aligned}
$$

which is a Hilbert space endowed with inner product and norm

$$
\begin{aligned}
(u, v)_{\mu, V} & =\int_{0}^{\infty} \mu(r)(u(r), v(r))_{V} d r \\
\|u\|_{\mu, V}^{2} & =(u, u)_{\mu, V}=\int_{0}^{\infty} \mu(r)\|u(r)\|_{V}^{2} d r .
\end{aligned}
$$

Finally, we introduce the phase space

$$
\mathscr{H}=V \times H \times L_{\mu}^{2}\left(\mathbb{R}^{+} ; V\right),
$$

equipped with the norm

$$
\|(u, v, \xi)\|_{\mathscr{H}}^{2}=\|\Delta u\|_{2}^{2}+\|v\|_{2}^{2}+\|\xi\|_{\mu, V}^{2} .
$$

In order to obtain the global attractor of problems (4)-(6), we need the following theorem. Under our hypotheses, we can derive an existence result by standard Faedo-Galerkin method (see [12, 28, 29]). For arguments involving the memory term, we follow Giorgi et al. [19, 20].

Theorem 1. Let (8)-(15) hold. If $h \in L^{2}(\Omega)$, then the following results hold.

(i) Provided that the initial data $\left(u_{0}, u_{1}, \eta_{0}\right) \in \mathscr{H}$, then problems (4)-(6) have a weak solution

$$
\left(u, u_{t}, \eta\right) \in C([0, T] ; \mathscr{H}), \quad \forall T>0,
$$

satisfying

$$
\begin{aligned}
& u \in L^{\infty}(0, T ; V), \\
& u_{t} \in L^{\infty}(0, T ; H), \\
& \eta \in L^{\infty}\left(0, T ; L_{\mu}^{2}\left(\mathbb{R}^{+} ; V\right)\right) .
\end{aligned}
$$

(ii) Assume that $z_{i}=\left(u^{i}, u_{t}^{i}, \eta^{i}\right)$ are weak solutions of problems (4)-(6) corresponding to initial data $z_{i}(0)=$ $\left(u_{0}^{i}, u_{1}^{i}, \eta_{0}^{i}\right), i=1,2$. Then,

$$
\begin{aligned}
& \left\|z_{1}(t)-z_{2}(t)\right\|_{\mathscr{H}} \leq e^{c t}\left\|z_{1}(0)-z_{2}(0)\right\|_{\mathscr{H}}, \\
& t \in[0, T],
\end{aligned}
$$

for some constant $c>0$.
Remark 2. Let $\left(u(t), u_{t}(t), \eta^{t}\right)$ be the unique weak solution of problems (4)-(6); by Theorem 1 , we can define a semigroup $S(t): \mathscr{H} \rightarrow \mathscr{H}$ as follows:

$$
S(t)\left(u_{0}, u_{1}, \eta_{0}\right)=\left(u(t), u_{t}(t), \eta^{t}\right), \quad t \geq 0 .
$$

And $S(t)$ is continuous on $\mathscr{H}$.

\section{Some Abstract Results}

In this section, we will recall some basic theories of infinite dimensional dynamical systems; we refer to [30] for more details.

Definition 3. The global attractor $\mathscr{A}$ is the maximal compact invariant set

$$
S(t) \mathscr{A}=\mathscr{A} \quad \forall t \geq 0
$$

and the minimal set that attracts all bounded sets:

$$
\lim _{t \rightarrow \infty} \operatorname{dist}_{\mathscr{H}}(S(t) B, \mathscr{A})=0,
$$

for any bounded set $B \subset \mathscr{H}$, where dist $_{\mathscr{H}}$ is the Hausdorff semidistance in $\mathscr{H}$.

Definition 4. A semigroup is dissipative if it possesses a bounded absorbing set; that is, for any bounded set $\mathscr{B} \subset \mathscr{H}$, there exists $t_{0}(B)$ such that

$$
S(t) B \subset \mathscr{B}, \quad \forall t \geq t_{0}(B) .
$$

Definition 5. $(\mathscr{H}, S(t))$ is asymptotically smooth if, for any bounded positively invariant set $B \subset \mathscr{H}$, there exists a compact set $K \subset \bar{B}$ such that

$$
\lim _{t \rightarrow \infty} \operatorname{dist}_{\mathscr{H}}(S(t) B, K)=0 .
$$

Theorem 6. A dissipative dynamical system $(\mathscr{H}, S(t))$ has a compact global attractor if and only if it is asymptotically smooth.

Theorem 7. For any bounded positively invariant set $B \subset \mathscr{H}$, $S(t)$ is called asymptotically smooth in $\mathscr{H}$, if, for any $\varepsilon>0$ and any sequence $\left\{z_{n}\right\}$ in $B$, there exists $T=T(\varepsilon, B)$ such that

$$
\|S(T) x-S(T) y\|_{\mathscr{H}} \leq \varepsilon+\phi_{T}(x, y), \quad \forall x, y \in B,
$$

where $\phi_{T}: B \times B \rightarrow \mathbb{R}$ satisfies

$$
\liminf _{n \rightarrow \infty} \liminf _{m \rightarrow \infty} \phi_{T}\left(z_{n}, z_{m}\right)=0 \text {. }
$$

\section{Existence of Attractors}

In this section, we will use the abstract results presented in Section 3 to prove our main result.

Lemma 8. Under assumptions (8)-(15), the semigroup $\{S(t)\}_{t \geq 0}$ corresponding to problems (4)-(6) has a bounded absorbing set in $\mathscr{H}$. 
Proof. Taking the scalar product in $H$ of (4) with $u_{t}$, we infer that

$$
\begin{aligned}
& \frac{d}{d t}\left[\frac{1}{2}\left\|u_{t}\right\|_{2}^{2}+\frac{1}{2}\|\Delta u\|_{2}^{2}+\frac{\lambda}{2}\|u\|_{2}^{2}\right. \\
& \left.\quad+\int_{\Omega}(F(u)-h u) d x\right]+\left(\eta, u_{t}\right)_{\mu, V} \\
& \quad+\int_{\Omega} g\left(u_{t}\right) u_{t} d x+\left(\left(p-\beta\|\nabla u\|_{2}^{2}\right) \Delta u, u_{t}\right)=0,
\end{aligned}
$$

where $F(u)=\int_{0}^{u} f(\tau) d \tau$.

Thanks to (5), (13), and (15) and Hölder inequality, we get

$$
\begin{aligned}
& \left(\eta, u_{t}\right)_{\mu, V}=\left(\eta, \eta_{t}+\eta_{s}\right)_{\mu, V} \\
& =\frac{1}{2} \frac{d}{d t}\|\eta\|_{\mu, V}^{2}+\int_{0}^{\infty} \mu(s)\left(\eta(s), \eta_{s}(s)\right)_{V} d s \\
& =\frac{1}{2} \frac{d}{d t}\|\eta\|_{\mu, V}^{2}+\frac{1}{2} \int_{0}^{\infty} \mu(s) d\|\eta(s)\|_{V}^{2} \\
& =\frac{1}{2} \frac{d}{d t}\|\eta\|_{\mu, V}^{2}-\frac{1}{2} \int_{0}^{\infty} \mu^{\prime}(s)\|\eta(s)\|_{V}^{2} d s \\
& \geq \frac{1}{2} \frac{d}{d t}\|\eta\|_{\mu, V}^{2}+\frac{k_{2}}{2} \int_{0}^{\infty} \mu(s)\|\eta(s)\|_{V}^{2} d s \\
& =\frac{1}{2} \frac{d}{d t}\|\eta\|_{\mu, V}^{2}+\frac{k_{2}}{2}\|\eta\|_{\mu, V}^{2}, \\
& \left(\left(p-\beta\|\nabla u\|_{2}^{2}\right) \Delta u, u_{t}\right) \\
& \quad=\frac{1}{2} \frac{d}{d t}\left(\frac{\beta}{\sqrt{2 \beta}}\|\nabla u\|_{2}^{2}-\frac{p}{\sqrt{2 \beta}}\right)^{2} .
\end{aligned}
$$

We conclude from (32)-(34) that

$$
\frac{d}{d t} E(t)+\frac{k_{2}}{2}\|\eta\|_{\mu, V}^{2}+\int_{\Omega} g\left(u_{t}\right) u_{t} d x \leq 0
$$

where

$$
\begin{aligned}
E(t)= & \frac{1}{2}\left\|u_{t}\right\|_{2}^{2}+\frac{1}{2}\|\Delta u\|_{2}^{2}+\frac{\lambda}{2}\|u\|_{2}^{2} \\
& +\int_{\Omega}(F(u)-h u) d x+\frac{1}{2}\|\eta\|_{\mu, V}^{2} \\
& +\frac{1}{2}\left(\frac{\beta}{\sqrt{2 \beta}}\|\nabla u\|_{2}^{2}-\frac{p}{\sqrt{2 \beta}}\right)^{2} .
\end{aligned}
$$

Since

$$
g\left(u_{t}\right)=g\left(u_{t}\right)-g(0)=g^{\prime}\left(\theta u_{t}\right) u_{t}, \quad 0<\theta<1,
$$

from (11), we obtain

$$
\int_{\Omega} g\left(u_{t}\right) u_{t} d x \geq l\left\|u_{t}\right\|^{2} .
$$

Thus, (35) implies

$$
E(t) \leq E(0), \quad \forall t \geq 0 .
$$

It follows from (10) that there exist $\lambda^{\prime}\left(\lambda_{1}>\lambda^{\prime}>0\right)$ and $C_{0}>0$, such that

$$
\begin{gathered}
(f(u), u)>-\lambda^{\prime}\|u\|_{2}^{2}-C_{0} \text { meas }(\Omega), \\
\int_{\Omega} F(u) d x>-\frac{\lambda^{\prime}}{2}\|u\|_{2}^{2}-C_{0} \operatorname{meas}(\Omega) .
\end{gathered}
$$

Using (40) and Poincaré and Young inequalities, we end up with

$$
\begin{aligned}
E(0) \geq & E(t) \\
= & \frac{1}{2}\left\|u_{t}\right\|_{2}^{2}+\frac{1}{2}\|\Delta u\|_{2}^{2}+\frac{\lambda}{2}\|u\|_{2}^{2} \\
& +\int_{\Omega}(F(u)-h u) d x+\frac{1}{2}\|\eta\|_{\mu, V}^{2} \\
& +\frac{1}{2}\left(\frac{\beta}{\sqrt{2 \beta}}\|\nabla u\|_{2}^{2}-\frac{p}{\sqrt{2 \beta}}\right)^{2} \\
\geq & \frac{1}{2}\left\|u_{t}\right\|_{2}^{2}+\frac{1}{2}\|\Delta u\|_{2}^{2}+\frac{\lambda}{2}\|u\|_{2}^{2}-\frac{\lambda^{\prime}}{2}\|u\|_{2}^{2} \\
& +\frac{1}{2}\|\eta\|_{\mu, V}^{2}-C_{0} \text { meas }(\Omega)-\frac{1}{\lambda}\|h\|^{2}-\frac{\lambda}{4}\|u\|_{2}^{2} \\
\geq & \frac{1}{2}\left\|u_{t}\right\|_{2}^{2}+C\|\Delta u\|_{2}^{2}+\frac{1}{2}\|\eta\|_{\mu, V}^{2} \\
& -C_{1}\left(\operatorname{meas}(\Omega)+\|h\|^{2}\right) \\
\geq & -C_{1}\left(\operatorname{meas}(\Omega)+\|h\|^{2}\right),
\end{aligned}
$$

where $C=1 / 2-\lambda^{\prime} / 2 \lambda_{1}, C_{1}=\max \left\{C_{0}, 1 / \lambda\right\}$.

By (35) and (41), we deduce that

$$
\begin{aligned}
\int_{0}^{t} \int_{\Omega} g\left(u_{t}\right) u_{t} d x d s \leq & -\int_{0}^{t} \frac{d}{d t} E(s) d s \\
= & E(0)-E(t) \\
\leq & E(0) \\
& +C_{1}\left(\operatorname{meas}(\Omega)+\|h\|^{2}\right), \\
& \forall t \geq 0 .
\end{aligned}
$$

Now, we set $v=u_{t}+\delta u$ and rewrite the equation of (4) as follows:

$$
\begin{gathered}
v_{t}-\delta u_{t}+g\left(u_{t}\right)+\Delta^{2} u+\int_{0}^{\infty} \mu(s) \Delta^{2} \eta^{t}(s) d s+\lambda u \\
+\left(p-\beta\|\nabla u\|_{2}^{2}\right) \Delta u+f(u)=h(x)
\end{gathered}
$$


We formally take the scalar product in $H$ of (43) with $v$; after a computation, we find

$$
\begin{aligned}
& \frac{d}{d t}\left[\frac{1}{2}\|v\|_{2}^{2}+\frac{1}{2}\|\Delta u\|_{2}^{2}+\frac{\lambda}{2}\|u\|_{2}^{2}-\frac{\delta^{2}}{2}\|u\|_{2}^{2}\right. \\
& \left.\quad+\int_{\Omega}(F(u)-h u) d x\right]+\left(g\left(u_{t}\right)-\delta u_{t}, u_{t}\right) \\
& \quad+\delta\left(g\left(u_{t}\right), u\right)+\delta\|\Delta u\|_{2}^{2}+\left(\left(p-\beta\|\nabla u\|_{2}^{2}\right) \Delta u, v\right) \\
& \quad+\left(\eta, u_{t}\right)_{\mu, V}+\delta(\eta, u)_{\mu, V}+\lambda \delta\|u\|_{2}^{2}+\delta(f(u), u) \\
& \quad-\delta(h, u)=0
\end{aligned}
$$

where

$$
\begin{aligned}
\left(\left(p-\beta\|\nabla u\|_{2}^{2}\right) \Delta u, v\right) \\
=\left(\left(p-\beta\|\nabla u\|_{2}^{2}\right) \Delta u, u_{t}+\delta u\right) \\
=\left(p-\beta\|\nabla u\|_{2}^{2}\right)\left(\Delta u, u_{t}\right) \\
\quad+\left(p-\beta\|\nabla u\|_{2}^{2}\right)(\Delta u, \delta u) \\
\geq \frac{1}{2} \frac{d}{d t}\left(\frac{\beta}{\sqrt{2 \beta}}\|\nabla u\|_{2}^{2}-\frac{p}{\sqrt{2 \beta}}\right)^{2} \\
\quad+\delta\left(\frac{\beta}{\sqrt{2 \beta}}\|\nabla u\|_{2}^{2}-\frac{p}{\sqrt{2 \beta}}\right)^{2}-\frac{\delta p^{2}}{2 \beta} .
\end{aligned}
$$

Denote

$$
\begin{aligned}
E_{\delta}(t)= & \frac{1}{2}\|v\|_{2}^{2}+\frac{1}{2}\|\Delta u\|_{2}^{2}+\frac{\lambda}{2}\|u\|_{2}^{2}-\frac{\delta^{2}}{2}\|u\|_{2}^{2} \\
& +\int_{\Omega}(F(u)-h u) d x+\frac{1}{2}\|\eta\|_{\mu, V}^{2} \\
& +\frac{1}{2}\left(\frac{\beta}{\sqrt{2 \beta}}\|\nabla u\|_{2}^{2}-\frac{p}{\sqrt{2 \beta}}\right)^{2}, \\
H(t)= & \left(g\left(u_{t}\right)-\delta u_{t}, u_{t}\right)+\delta\left(g\left(u_{t}\right), u\right)+\delta\|\Delta u\|_{2}^{2} \\
& +\frac{k_{2}}{2}\|\eta\|_{\mu, V}^{2}+\delta\left(\frac{\beta}{\sqrt{2 \beta}}\|\nabla u\|_{2}^{2}-\frac{p}{\sqrt{2 \beta}}\right)^{2} \\
& -\frac{\delta p^{2}}{2 \beta}+\delta(\eta, u)_{\mu, V}+\lambda \delta\|u\|_{2}^{2} \\
& +\delta(f(u), u)-\delta(h, u) .
\end{aligned}
$$

Therefore, together with (33) and (44), we get

$$
\frac{d}{d t} E_{\delta}(t)+H(t) \leq 0
$$

From (40), for any $\delta$ sufficiently small, using Hölder and Young inequalities, we get

$$
\begin{aligned}
E_{\delta}(t) \geq & \frac{1}{2}\|v\|_{2}^{2}+C\|\Delta u\|_{2}^{2}+\frac{1}{2}\|\eta\|_{\mu, V}^{2} \\
& -C_{1}\left(\operatorname{meas}(\Omega)+\|h\|^{2}\right) .
\end{aligned}
$$

Thanks to (11), we get

$$
\left(g\left(u_{t}\right)-\delta u_{t}, u_{t}\right) \geq(l-\delta)\left\|u_{t}\right\|^{2} .
$$

Using the Young inequality, we obtain

$$
\delta(\eta, u)_{\mu, V} \geq-\frac{k_{2}}{4}\|\eta\|_{\mu, V}^{2}-\frac{\mu_{0} \delta^{2}}{k_{2}}\|\Delta u\|_{2}^{2} .
$$

From (11) and (12), we deduce that

$$
\begin{aligned}
|g(s)|^{(q+1) / q} & =|g(s)|^{1 / q} \cdot|g(s)| \leq C_{2}(1+|s|)|g(s)| \\
& \leq \begin{cases}C_{2}, & |s| \leq 1, \\
2 C_{2} g(s) s, & |s| \geq 1,\end{cases}
\end{aligned}
$$

where $C_{2}$ is a constant which is independent of $s$.

In line with (52), the Hölder and Young inequalities, and the Sobolev embedding $H_{0}^{2} \hookrightarrow L^{q+1}$, similar to the progress of [31], we conclude

$$
\left|\int_{\Omega} g\left(u_{t}\right) u d x\right| \leq \int_{\Omega\left(\left|u_{t}\right| \leq 1\right)}\left|g\left(u_{t}\right) u\right| d x
$$

$$
\begin{aligned}
& +\int_{\Omega\left(\left|u_{t}\right| \geq 1\right)}\left|g\left(u_{t}\right) u\right| d x \leq \int_{\Omega\left(\left|u_{t}\right| \leq 1\right)} C_{2}|u| d x \\
& +\left(\int_{\Omega\left(\left|u_{t}\right| \geq 1\right)}\left|g\left(u_{t}\right)\right|^{(q+1) / q} d x\right)^{q /(q+1)} \\
& \cdot\left(\int_{\Omega\left(\left|u_{t}\right| \geq 1\right)}|u|^{q+1} d x\right)^{1 /(q+1)} \leq \int_{\Omega\left(\left|u_{t}\right| \leq 1\right)} C_{2}|u| d x \\
& +2 C_{2}\left(\int_{\Omega\left(\left|u_{t}\right| \geq 1\right)} g\left(u_{t}\right) u_{t} d x\right)^{q /(q+1)} \\
& \cdot\left(\int_{\Omega\left(\left|u_{t}\right| \geq 1\right)}|u|^{q+1} d x\right)^{1 /(q+1)} \leq \frac{C_{2}}{4 \varepsilon} \int_{\Omega} d x \\
& +C_{2} \varepsilon\|u\|^{2}+C_{\varepsilon}\left(\int_{\Omega\left(\left|u_{t}\right| \geq 1\right)} g\left(u_{t}\right) u_{t} d x\right) \\
& \cdot\|u\|_{q+1}^{(q-1) / q}+\varepsilon\|u\|_{q+1}^{2} \leq \frac{C_{2}}{4 \varepsilon} \operatorname{meas}(\Omega)+C_{2} \varepsilon\|u\|^{2} \\
& +C_{s} C_{\varepsilon}\|\Delta u\|_{2}^{(q-1) / q} \int_{\Omega} g\left(u_{t}\right) u_{t} d x+\varepsilon C_{s}\|\Delta u\|_{2}^{2},
\end{aligned}
$$

where $\varepsilon$ is a small positive constant and $C_{s}$ is an embedding constant. 
Therefore, we choose $\varepsilon$ and $\delta$ small enough, such that

$$
\begin{aligned}
\frac{\lambda}{2}-C_{2} \varepsilon & >0, \\
2 C-\frac{\mu_{0} \delta}{k_{2}}-\varepsilon C_{s} & >\frac{C}{4}, \\
l-\delta & >\frac{l}{4} .
\end{aligned}
$$

Together with (40), (50), (51), and (53), we have

$$
\begin{aligned}
H( & t) \geq(l-\delta)\left\|u_{t}\right\|^{2}+\delta\|\Delta u\|_{2}^{2}+\frac{k_{2}}{4}\|\eta\|_{\mu, V}^{2} \\
- & \frac{\mu_{0} \delta^{2}}{k_{2}}\|\Delta u\|_{2}^{2}+\delta\left(\frac{\beta}{\sqrt{2 \beta}}\|\nabla u\|_{2}^{2}-\frac{p}{\sqrt{2 \beta}}\right)^{2}-\frac{\delta p^{2}}{2 \beta} \\
+ & \lambda \delta\|u\|_{2}^{2}-\delta \lambda^{\prime}\|u\|_{2}^{2}-\delta C_{0} \operatorname{meas}(\Omega)-\frac{\delta \lambda}{2}\|u\|^{2} \\
& -\frac{\delta}{2 \lambda}\|h\|^{2}-\delta\left(\frac{C_{2}}{4 \varepsilon} \operatorname{meas}(\Omega)+C_{2} \varepsilon\|u\|^{2}\right. \\
+ & \left.C_{s} C_{\varepsilon}\|\Delta u\|_{2}^{(q-1) / q} \int_{\Omega} g\left(u_{t}\right) u_{t} d x+\varepsilon C_{s}\|\Delta u\|_{2}^{2}\right) \\
& \geq(l-\delta)\left\|u_{t}\right\|^{2}-C_{\delta}^{\prime}\left(\operatorname{meas}(\Omega)+\|h\|^{2}\right)+\delta\left(\frac{\lambda}{2}\right. \\
& \left.-C_{2} \varepsilon\right)\|u\|^{2}+\delta\left(2 C-\frac{\mu_{0} \delta}{k_{2}}-\varepsilon C_{s}\right)\|\Delta u\|^{2} \\
+ & \frac{k_{2}}{4}\|\eta\|_{\mu, V}^{2}-\delta C_{s} C_{\varepsilon}\|\Delta u\|_{2}^{(q-1) / q} \int_{\Omega} g\left(u_{t}\right) u_{t} d x \\
+ & \frac{\delta p^{2}}{2 \beta} \geq \frac{l}{4}\left\|u_{t}\right\|^{2}-C_{\delta}^{\prime}\left(\operatorname{meas}(\Omega)+\|h\|^{2}\right) \\
& \frac{\delta C}{4}, k^{2}+\frac{k_{2}}{4}\|\eta\|_{\mu, V}^{2}-C_{E(0)} g\left(u_{t}\right) u_{t} d x
\end{aligned}
$$

where $C_{E(0)}$ is a constant which depends on $\delta, C_{s}, C_{\varepsilon}$, and $E(0)$, while $C_{\delta}^{\prime}$ is a constant which depends on $\delta, C_{0}, \lambda, C_{2}$, and $\varepsilon$.

Notice that

$$
\begin{aligned}
& \left\|u_{t}\right\|^{2}+\|\Delta u\|^{2}+\|\eta\|_{\mu, V}^{2} \\
& =\left\|u_{t}+\delta u-\delta u\right\|^{2}+\|\Delta u\|^{2}+\|\eta\|_{\mu, V}^{2} \\
& =\|v-\delta u\|^{2}+\|\Delta u\|^{2}+\|\eta\|_{\mu, V}^{2} \\
& \leq 2\|v\|^{2}+2 \delta^{2}\|u\|^{2}+\|\Delta u\|^{2}+\|\eta\|_{\mu, V}^{2} \\
& \leq 2\|v\|^{2}+\left(\frac{2 \delta^{2}}{\lambda_{1}}+1\right)\|\Delta u\|^{2}+\|\eta\|_{\mu, V}^{2} \\
& \leq c_{0}\left(\|v\|^{2}+\|\Delta u\|^{2}+\|\eta\|_{\mu, V}^{2}\right),
\end{aligned}
$$

where $c_{0}=\max \left\{2,1+2 \delta^{2} \lambda_{1}^{-1}\right\}$.
Integrating (48) from 0 to $t$, combining with (42), (49), and (55), we arrive at

$$
\begin{gathered}
\left\|u_{t}\right\|^{2}+\|\Delta u\|^{2}+\|\eta\|_{\mu, V}^{2}-\frac{4 c_{0}}{C}\left[C_{1}\left(\operatorname{meas}(\Omega)+\|h\|^{2}\right)\right. \\
\left.+E_{\delta(0)}+C_{E(0)}\left(E(0)+C_{1}\left(\operatorname{meas}(\Omega)+\|h\|^{2}\right)\right)\right] \\
\leq-\int_{0}^{t}\left(\frac{\delta^{\prime} c_{0}}{C}\left(\left\|u_{t}(s)\right\|^{2}+\|\Delta u(s)\|^{2}+\left\|\eta^{s}(\tau)\right\|_{\mu, V}^{2}\right)\right. \\
\left.-\frac{4 c_{0}}{C} C_{\delta}^{\prime}\left(\operatorname{meas}(\Omega)+\|h\|^{2}\right)-\frac{4 c_{0}}{C} \cdot \frac{\delta p^{2}}{2 \beta}\right) d s,
\end{gathered}
$$

where $\delta^{\prime}=\min \left\{l, \delta C, k_{2}\right\}$.

Therefore, for any $r>\left(4 C_{\delta}^{\prime} \beta\left(\operatorname{meas}(\Omega)+\|h\|^{2}\right)+2 \delta p^{2}\right) / \delta^{\prime} \beta$, there exists $t_{0}>0$, such that

$$
\left\|u_{t}\left(t_{0}\right)\right\|^{2}+\left\|\Delta u\left(t_{0}\right)\right\|^{2}+\left\|\eta^{t_{0}}(s)\right\|_{\mu, V}^{2} \leq r
$$

Denote

$$
\begin{aligned}
& B_{0} \\
& =\left\{\left(u_{0}, v_{0}, \eta_{0}\right) \in \mathscr{H}:\left\|\Delta u_{0}\right\|^{2}+\left\|v_{0}\right\|^{2}+\left\|\eta_{0}\right\|_{\mu, V}^{2} \leq r\right\} ;
\end{aligned}
$$

by the argument above, we know that $B_{0}$ is a bounded absorbing set.

Define

$$
B=\bigcup_{t \geq 0} S(t) B_{0}
$$

then $B$ is also a bounded absorbing set. This shows that the semigroup $\{S(t)\}_{t \geq 0}$ corresponding to problems (4)-(6) has a bounded absorbing set in $\mathscr{H}$.

Remark 9. If $\left(u, u_{t}, \eta\right)$ is a solution of (4)-(6) with initial data $\left(u_{0}, u_{1}, \eta_{0}\right)$ in a bounded set $B$, then we have

$$
\left\|\left(u(t), u_{t}(t), \eta^{t}\right)\right\|_{\mathscr{H}} \leq C_{B}, \quad \forall t \geq 0,
$$

where $C_{B}>0$ is a constant depending on $B$.

In order to prove that the dynamical system $(\mathscr{H}, S(t))$ is asymptotically smooth, we need the following lemma.

Lemma 10. Assume that assumptions (8)-(15) hold and $g \in$ $L^{2}(\Omega) ; B \subset \mathscr{H}$ is a bounded set; let $z_{1}=\left(u, u_{t}, \eta\right)$ and $z_{2}=$ $\left(v, v_{t}, \xi\right)$ be two solutions of problems $(4)-(6)$, such that $z_{1}(0)=$ $\left(u_{0}, u_{1}, \eta_{0}\right)$ and $z_{2}(0)=\left(v_{0}, v_{1}, \xi_{0}\right)$ are in $B$. Then,

$$
\begin{aligned}
& \left\|z_{1}(t)-z_{2}(t)\right\|_{\mathscr{H}}^{2} \\
& \leq C^{*} e^{-\alpha_{2} t} \\
& \quad+C^{*} \int_{0}^{t} e^{-\alpha_{2}(t-s)}\left(\left\|w_{t}(s)\right\|_{2}^{2}+\|w(s)\|_{2(\rho+1)}^{2}\right) d s,
\end{aligned}
$$

for any $t \geq 0$, where $C^{*}>0$ and $\alpha_{2}>0$ are constants. 
Proof. For convenience, we denote $w=u-v$ and $\zeta=\eta-\xi$. Then, $(w, \zeta)$ satisfy the following equations:

$$
\begin{aligned}
w_{t t} & +g\left(u_{t}\right)-g\left(v_{t}\right)+\Delta^{2} w+\int_{0}^{\infty} \mu(s) \Delta^{2} \zeta^{t}(s) d s \\
& +\lambda w+\left(p-\beta\|\nabla u\|_{2}^{2}\right) \Delta u-\left(p-\beta\|\nabla v\|_{2}^{2}\right) \Delta v \\
& +f(u)-f(v)=0, \\
\zeta_{t} & =-\zeta_{s}+w_{t},
\end{aligned}
$$

and initial datum

$$
\begin{gathered}
w(0)=u_{0}-v_{0}, \\
w_{t}(0)=u_{1}-v_{1}, \\
\zeta^{0}=\eta_{0}-\xi_{0} .
\end{gathered}
$$

Taking the scalar product in $H$ of (63) with $\varphi=w_{t}+\sigma w$, we obtain

$$
\begin{aligned}
\frac{d}{d t}\left(\frac{1}{2}\right. & \left.\|\varphi\|_{2}^{2}+\frac{1}{2}\|\Delta w\|_{2}^{2}\right) \\
& +\left(g^{\prime}\left(v_{t}+\theta\left(u_{t}-v_{t}\right)\right)-\sigma\right)\left(w_{t}, \varphi\right)+\sigma\|\Delta w\|_{2}^{2} \\
& +\left(\left(p-\beta\|\nabla u\|_{2}^{2}\right) \Delta u, \varphi\right) \\
& -\left(\left(p-\beta\|\nabla v\|_{2}^{2}\right) \Delta v, \varphi\right)+\lambda(w, \varphi)+\left(\zeta, w_{t}\right)_{\mu, V} \\
& +\sigma(\zeta, w)_{\mu, V}+(f(u)-f(v), \varphi)=0,
\end{aligned}
$$

where $0<\theta<1$.

Noting the similar estimate used in Lemma 8, we obtain

$$
\begin{aligned}
& \left(\left(g^{\prime}\left(v_{t}+\theta\left(u_{t}-v_{t}\right)\right)-\sigma\right) w_{t}, \varphi\right) \geq(l-\sigma)\left(w_{t}, \varphi\right) \\
& \geq(l-\sigma)\|\varphi\|_{2}^{2}-\sigma(l-\sigma)(w, \varphi) \\
& \left(\zeta, w_{t}\right)_{\mu, V} \geq \frac{1}{2} \frac{d}{d t}\|\zeta\|_{\mu, V}^{2}+\frac{k_{2}}{2}\|\zeta\|_{\mu, V}^{2}, \\
& \sigma(\zeta, w)_{\mu, V} \geq-\frac{k_{2}}{4}\|\zeta\|_{\mu, V}^{2}-\frac{\mu_{0} \sigma^{2}}{k_{2}}\|\Delta w\|_{2}^{2} .
\end{aligned}
$$

Using the Poincaré, Hölder, and Young inequalities and taking $\sigma$ small enough, such that

$$
\begin{array}{r}
\frac{l}{2}-\sigma>\frac{l}{4}, \\
1-\frac{\mu_{0} \sigma}{k_{2}}-\frac{\sigma l}{2 \lambda_{1}}>\frac{1}{2},
\end{array}
$$

then we obtain

$$
\begin{aligned}
(l-\sigma) & \|\varphi\|_{2}^{2}-\sigma(l-\sigma)(w, \varphi)+\left(\sigma-\frac{\mu_{0} \sigma^{2}}{k_{2}}\right)\|\Delta w\|_{2}^{2} \\
\geq & (l-\sigma)\|\varphi\|_{2}^{2}-\left(\frac{\sigma^{2} l}{2 \lambda_{1}}\|\Delta w\|_{2}^{2}+\frac{l}{2}\|\varphi\|_{2}^{2}\right) \\
& +\sigma\left(1-\frac{\mu_{0} \sigma}{k_{2}}\right)\|\Delta w\|_{2}^{2} \\
\geq & \left(\frac{l}{2}-\sigma\right)\|\varphi\|_{2}^{2}+\sigma\left(1-\frac{\mu_{0} \sigma}{k_{2}}-\frac{\sigma l}{2 \lambda_{1}}\right)\|\Delta w\|_{2}^{2} \\
\geq & \frac{l}{4}\|\varphi\|_{2}^{2}+\frac{\sigma}{2}\|\Delta w\|_{2}^{2} .
\end{aligned}
$$

Combining (69) with (66), we get

$$
\begin{aligned}
\frac{d}{d t}\left(\frac{1}{2}\|\varphi\|_{2}^{2}+\frac{1}{2}\|\Delta w\|_{2}^{2}+\frac{1}{2}\|\zeta\|_{\mu, V}^{2}\right)+\frac{\sigma}{2}\|\Delta w\|_{2}^{2} \\
\quad+\frac{l}{4}\|\varphi\|_{2}^{2}+\frac{k_{2}}{4}\|\zeta\|_{\mu, V}^{2}+\left(\left(p-\beta\|\nabla u\|_{2}^{2}\right) \Delta u, \varphi\right) \\
\quad-\left(\left(p-\beta\|\nabla v\|_{2}^{2}\right) \Delta v, \varphi\right) \\
\leq-\lambda(w, \varphi)-(f(u)-f(v), \varphi)
\end{aligned}
$$

besides,

$$
\begin{aligned}
|-\lambda(w, \varphi)| & \leq \frac{\lambda^{2}}{\sigma}\|w\|_{2}^{2}+\frac{\sigma}{4}\|\varphi\|_{2}^{2} \\
& \leq \frac{\lambda^{2} c_{1}}{\sigma}\|w\|_{2(\rho+1)}^{2}+\frac{\sigma}{4}\|\varphi\|_{2}^{2},
\end{aligned}
$$

where $c_{1}>0$ is an embedding constant for $L^{2(\rho+1)}(\Omega) \hookrightarrow$ $L^{2}(\Omega)$.

By virtue of the generalized Hölder inequality with $\rho / 2(\rho+1)+1 / 2(\rho+1)+1 / 2=1,(8),(61)$, and Young inequality, we have

$$
\begin{aligned}
& \left|-\int_{\Omega}(f(u(t))-f(v(t))) \varphi(t) d x\right| \\
& \quad \leq k_{0} \int_{\Omega}\left(1+|u(t)|^{\rho}+|v(t)|^{\rho}\right)|w(t)||\varphi(t)| d x \\
& \quad \leq k_{0}\left(|\Omega|^{\rho / 2(\rho+1)}+\|u\|_{2(\rho+1)}^{\rho}+\|v\|_{2(\rho+1)}^{\rho}\right)\|w\|_{2(\rho+1)} \\
& \cdot\|\varphi\|_{2} \leq C_{B}\|w\|_{2(\rho+1)}\|\varphi\|_{2} \leq \frac{C_{B}^{2}}{\sigma}\|w\|_{2(\rho+1)}^{2} \\
& \quad+\frac{\sigma}{4}\|\varphi\|_{2}^{2} .
\end{aligned}
$$

Now, we estimate $\left(\left(p-\beta\|\nabla u\|_{2}^{2}\right) \Delta u, \varphi\right)-\left(\left(p-\beta\|\nabla v\|_{2}^{2}\right) \Delta v, \varphi\right)$. Set

$$
\begin{aligned}
& \left(\left(p-\beta\|\nabla u\|_{2}^{2}\right) \Delta u, \varphi\right)-\left(\left(p-\beta\|\nabla v\|_{2}^{2}\right) \Delta v, \varphi\right) \\
& \quad=\int_{\Omega}\left[\left(p-\beta\|\nabla u\|_{2}^{2}\right) \Delta u-\left(p-\beta\|\nabla v\|_{2}^{2}\right) \Delta v\right] \\
& \quad \cdot \varphi(t) d x=I_{1}+I_{2},
\end{aligned}
$$


where

$$
\begin{aligned}
& I_{1}=\int_{\Omega}(p \Delta u-p \Delta v) \varphi(t) d x \\
& I_{2}=\int_{\Omega}\left(-\beta\|\nabla u\|_{2}^{2} \Delta u+\beta\|\nabla v\|_{2}^{2} \Delta v\right) \varphi(t) d x .
\end{aligned}
$$

Taking advantage of the Hölder and Young inequalities, (61), it follows that

$$
\begin{aligned}
& \left|I_{1}\right|=\left|\int_{\Omega}(p \Delta u-p \Delta v) \varphi(t) d x\right| \leq \int_{\Omega}|p||\Delta w| \\
& \cdot|\varphi(t)| d x \leq \sigma^{2} p^{2}\|\Delta w\|_{2}^{2}+\frac{1}{4 \sigma^{2}}\|\varphi\|_{2}^{2} \\
& \leq \sigma^{2} p^{2}\|\Delta w\|_{2}^{2}+\frac{1}{4 \sigma^{2}}\left\|w_{t}+\sigma w\right\|_{2}^{2} \leq \sigma^{2} p^{2}\|\Delta w\|_{2}^{2} \\
& +\frac{1}{2 \sigma^{2}}\left\|w_{t}\right\|_{2}^{2}+\frac{1}{2}\|w\|_{2}^{2} \leq \sigma^{2} p^{2}\|\Delta w\|_{2}^{2}+\frac{1}{2 \sigma^{2}}\left\|w_{t}\right\|_{2}^{2} \\
& +\frac{c_{1}}{2}\|w\|_{2(\rho+1)}^{2} \text {, } \\
& \left|I_{2}\right|=\left|\int_{\Omega}\left(\beta\|\nabla u\|_{2}^{2} \Delta u-\beta\|\nabla v\|_{2}^{2} \Delta v\right) \varphi(t) d x\right| \\
& \leq \int_{\Omega} \mid\left(\beta\|\nabla u\|_{2}^{2} \Delta u-\beta\|\nabla u\|_{2}^{2} \Delta v+\beta\|\nabla u\|_{2}^{2} \Delta v\right. \\
& \left.-\beta\|\nabla v\|_{2}^{2} \Delta v\right) \varphi(t)\left|d x \leq \beta \int_{\Omega}\|\nabla u\|_{2}^{2}\right| \Delta w \mid \\
& \cdot|\varphi(t)| d x+\beta \int_{\Omega}\left(\|\nabla u\|_{2}^{2}+\|\nabla v\|_{2}^{2}\right)|\Delta v| \\
& \cdot|\varphi(t)| d x \leq \frac{C_{B} \beta}{2} \int_{\Omega}|\Delta w||\varphi(t)| d x \\
& +\sqrt{\frac{C_{B}}{2}} \beta \int_{\Omega}|\Delta v||\varphi(t)| d x \leq \frac{C_{B} \beta}{2}\|\Delta w\|_{2}\|\varphi\|_{2} \\
& +\sqrt{\frac{C_{B}}{2}} \beta\|\Delta v\|_{2}\|\varphi\|_{2} \leq C_{B} \beta\left(\sigma^{2}\|\Delta w\|_{2}^{2}\right. \\
& \left.+\frac{1}{4 \sigma^{2}}\|\varphi\|_{2}^{2}\right) \leq C_{B} \beta \sigma^{2}\|\Delta w\|_{2}^{2}+\frac{C_{B} \beta}{2 \sigma^{2}}\left\|w_{t}\right\|_{2}^{2} \\
& +\frac{C_{B} \beta c_{1}}{2}\|w\|_{2(\rho+1)}^{2},
\end{aligned}
$$

where we have used the fact that $\|\Delta v\|_{2}=\|\Delta u-\Delta w\|_{2} \leq$ $\|\Delta u\|_{2}+\|\Delta w\|_{2} \leq \sqrt{C_{B} / 2}\|\Delta w\|_{2}$.

Plugging the above two inequalities into (73), we obtain

$$
\begin{aligned}
((p- & \left.\left.\beta\|\nabla u\|_{2}^{2}\right) \Delta u, \varphi\right)-\left(\left(p-\beta\|\nabla v\|_{2}^{2}\right) \Delta v, \varphi\right) \\
\geq & -\left(\sigma^{2} p^{2}+C_{B} \beta \sigma^{2}\right)\|\Delta w\|_{2}^{2} \\
& -\left(\frac{1}{2 \sigma^{2}}+\frac{C_{B} \beta}{2 \sigma^{2}}\right)\left\|w_{t}\right\|_{2}^{2} \\
& -\left(\frac{c_{1}}{2}+\frac{C_{B} \beta c_{1}}{2}\right)\|w\|_{2(\rho+1)}^{2} .
\end{aligned}
$$

Combining with (71), (73), and (76), we deduce from (70)

$$
\begin{aligned}
\frac{d}{d t}\left(\frac{1}{2}\|\varphi\|_{2}^{2}+\frac{1}{2}\|\Delta w\|_{2}^{2}+\frac{1}{2}\|\zeta\|_{\mu, V}^{2}\right) \\
+\left[\frac{\sigma}{2}-\sigma^{2} p^{2}-C_{B} \beta \sigma^{2}\right]\|\Delta w\|_{2}^{2} \\
+\left(\frac{l}{4}-\frac{\sigma}{2}\right)\|\varphi\|_{2}^{2}+\frac{k_{2}}{4}\|\zeta\|_{\mu, V}^{2} \\
\leq\left(\frac{1}{2 \sigma^{2}}+\frac{C_{B} \beta}{2 \sigma^{2}}\right)\left\|w_{t}\right\|_{2}^{2} \\
+\left(\frac{\lambda^{2} c_{1}}{\sigma}+\frac{C_{B}^{2}}{\sigma}+\frac{c_{1}}{2}+\frac{C_{B} \beta c_{1}}{2}\right)\|w\|_{2(\rho+1)}^{2} .
\end{aligned}
$$

We can choose $\sigma$ so small such that

$$
\begin{aligned}
\frac{\sigma}{2}-\sigma^{2} p^{2}-C_{B} \beta \sigma^{2}=\sigma\left(\frac{1}{2}-\sigma p^{2}-C_{B} \beta \sigma\right) & >0, \\
& \frac{l}{4}-\frac{\sigma}{2}>0 .
\end{aligned}
$$

Then, we set

$$
\begin{aligned}
E_{W}(t) & =\|\varphi\|_{2}^{2}+\|\Delta w\|_{2}^{2}+\|\zeta\|_{\mu, V}^{2}, \\
\frac{d}{d t} E_{W}(t)+\alpha_{2} E_{W}(t) & \leq C_{3}\left(\left\|w_{t}\right\|_{2}^{2}+\|w\|_{2(\rho+1)}^{2}\right),
\end{aligned}
$$

where

$$
\begin{aligned}
& \alpha_{2}=\min \left\{\sigma-2 \sigma^{2} p^{2}-2 C_{B} \beta \sigma^{2}, \frac{l}{2}-\sigma, \frac{k_{2}}{2}\right\}, \\
& C_{3}=\max \left\{\frac{1}{\sigma^{2}}+\frac{C_{B} \beta}{\sigma^{2}}, \frac{2 \lambda^{2} c_{1}}{\sigma}+\frac{2 C_{B}^{2}}{\sigma}+c_{1}+C_{B} \beta c_{1}\right\} .
\end{aligned}
$$

By the Gronwall lemma, we get

$$
\begin{aligned}
& E_{W}(t) \\
& \quad \leq E_{W}(0) e^{-\alpha_{2} t} \\
& \quad+C_{3} \int_{0}^{t} e^{-\alpha_{2}(t-s)}\left(\left\|w_{t}(s)\right\|_{2}^{2}+\|w(s)\|_{2(\rho+1)}^{2}\right) d s .
\end{aligned}
$$

Hence,

$$
\begin{aligned}
\left\|z_{1}(t)-z_{2}(t)\right\|_{\mathscr{H}}^{2}= & \|\Delta w\|_{2}^{2}+\left\|w_{t}\right\|_{2}^{2}+\|\zeta\|_{\mu, V}^{2} \\
= & \|\Delta w\|_{2}^{2}+\|\varphi-\sigma w\|_{2}^{2}+\|\zeta\|_{\mu, V}^{2} \\
\leq & \|\Delta w\|_{2}^{2}+2\|\varphi\|_{2}^{2}+2 \sigma^{2}\|w\|_{2}^{2} \\
& +\|\zeta\|_{\mu, V}^{2} \\
\leq & \left(1+\frac{2 \sigma^{2}}{\lambda_{1}}\right)\|\Delta w\|_{2}^{2}+2\|\varphi\|_{2}^{2} \\
& +\|\zeta\|_{\mu, V}^{2} \\
\leq & C_{4}\left(\|\varphi\|_{2}^{2}+\|\Delta w\|_{2}^{2}+\|\zeta\|_{\mu, V}^{2}\right),
\end{aligned}
$$

where $C_{4}=\max \left\{1+2 \sigma^{2} / \lambda_{1}, 2\right\}$. 
Namely,

$$
\begin{aligned}
& \left\|z_{1}(t)-z_{2}(t)\right\|_{\mathscr{H}}^{2} \leq C_{4} E_{W}(t) \\
& \quad \leq C_{4} E_{W}(0) e^{-\alpha_{2} t} \\
& \quad+C_{3} C_{4} \int_{0}^{t} e^{-\alpha_{2}(t-s)}\left(\left\|w_{t}(s)\right\|_{2}^{2}+\|w(s)\|_{2(\rho+1)}^{2}\right) d s .
\end{aligned}
$$

It is not difficult to know that (62) holds, where $C^{*}=$ $\max \left\{C_{4} E_{W}(0), C_{3} C_{4}\right\}$.

Now, we shall show that the dynamical system $(\mathscr{H}, S(t))$ is asymptotically smooth.

Lemma 11. Assume that assumptions (8)-(15) hold and $g \in$ $L^{2}(\Omega)$. Then, the dynamical system $(\mathscr{H}, S(t))$ is asymptotically smooth.

Proof. Given a bounded positively invariant set $B$ of $\mathscr{H}$, for $z_{1}, z_{2} \in B$, denote $S(t) z_{1}=\left(u(t), u_{t}(t), \eta^{t}\right)$ and $S(t) z_{2}=$ $\left(v(t), v_{t}(t), \xi^{t}\right)$ as the solutions of (4)-(6). Then, given $\varepsilon>0$, choosing $T>0$, such that $C^{*} e^{-\alpha_{2} T / 2}<\varepsilon$, we can infer from (62) that

$$
\begin{aligned}
& \left\|S(T) z_{1}-S(T) z_{2}\right\|_{\mathscr{H}} \leq \varepsilon \\
& \quad+C_{B}\left(\int _ { 0 } ^ { T } \left(\|u(s)-v(s)\|_{2(\rho+1)}^{2}\right.\right. \\
& \left.\left.\quad+\left\|u_{t}(s)-v_{t}(s)\right\|_{2}^{2}\right) d s\right)^{1 / 2}
\end{aligned}
$$

where $C_{B}$ is a constant which depends only on the size of $B$.

Now, we estimate the right hand side of (84). Condition (9) implies that $2<2(\rho+1)<\infty$ if $1 \leq N \leq 4$ and $2<2(\rho+$ $1) \leq 2 N /(N-4)$ if $N \geq 5$. Taking $\theta=(N / 4)(1-1 /(\rho+1))$ and applying the Galiardo-Nirenberg interpolation inequality, we have

$$
\begin{aligned}
& \|u(t)-v(t)\|_{2(\rho+1)} \\
& \quad \leq C_{\theta}\|\Delta(u(t)-v(t))\|_{2}^{\theta}\|u(t)-v(t)\|_{2}^{1-\theta} \\
& \quad \leq C_{B}\|u(t)-v(t)\|_{2}^{1-\theta} .
\end{aligned}
$$

Since $\|u(t)\|_{2}$ and $\|v(t)\|_{2}$ are uniformly bounded, there exists a constant $C_{B}>0$ such that

$$
\|u(t)-v(t)\|_{2(\rho+1)}^{2} \leq C_{B}\|u(t)-v(t)\|_{2}^{2(1-\theta)} .
$$

Using (86), we can rewrite (84) as

$$
\left\|S(T) z_{1}-S(T) z_{2}\right\|_{\mathscr{H}} \leq \varepsilon+\Phi_{T}\left(z_{1}, z_{2}\right),
$$

with

$$
\begin{aligned}
& \Phi_{T}\left(z_{1}, z_{2}\right)=C_{B}\left(\int _ { 0 } ^ { T } \left(C_{B}\|u(s)-v(s)\|_{2}^{2(1-\theta)}\right.\right. \\
& \left.\left.+\left\|u_{t}(s)-v_{t}(s)\right\|_{2}^{2}\right) d s\right)^{1 / 2} .
\end{aligned}
$$

We claim that $\Phi_{T}$ satisfies (31). In fact, let $z_{n} \in B$ with $z_{n}=$ $\left(u_{0}^{n}, u_{1}^{n}, \eta_{0}^{n}\right)$ be given. Denote $S(t) z_{n}=\left(u^{n}(t), u_{t}^{n}(t), \eta^{n, t}\right)$. It can be shown that $B$ is invariant by the property of $S(t)$, and hence $\left(u^{n}(t), u_{t}^{n}(t), \eta^{n, t}\right)$ are uniformly bounded in $\mathscr{H}$.

Particularly,

$$
\left(u^{n}, u_{t}^{n}\right) \text { is bounded in } C([0, T], V \times H), \quad T>0 .
$$

Then, by the fact $V \hookrightarrow H$ and the Aubins lemma, it is not hard to show that there exists a subsequence $\left\{u^{n_{k}}\right\}$ that converges strongly in $C([0, T], H)$; therefore,

$$
\lim _{k \rightarrow \infty} \lim _{l \rightarrow \infty} \int_{0}^{T}\left(C_{B}\left\|u^{n_{k}}(s)-u^{n_{l}}(s)\right\|_{2}^{2(1-\theta)}\right) d s=0 .
$$

Without loss of generality (at most by passing subsequences), we assume that

$$
u_{t}^{n} \longrightarrow u_{t} \text { weakly star in } L^{\infty}\left(0, T ; L^{2}(\Omega)\right)
$$

following similar argument given in [32, Lemma 4.4] and [31], we get

$$
\begin{gathered}
\lim _{k \rightarrow \infty} \lim _{l \rightarrow \infty} \int_{0}^{T}\left(C_{B}\left\|u^{n_{k}}(s)-u^{n_{l}}(s)\right\|_{2}^{2(1-\theta)}\right. \\
\left.+\left\|u_{t}^{n_{k}}(s)-u_{t}^{n_{l}}(s)\right\|_{2}^{2}\right) d s=0 ;
\end{gathered}
$$

then, the asymptotic smoothness property of $(\mathscr{H}, S(t))$ follows from Theorem 7 .

Finally, we obtain our main results.

Theorem 12. Assume that assumptions (8)-(15) hold; then, the dynamical system $(\mathscr{H}, S(t))$ corresponding to system (4)-(6) has a compact global attractor $\mathscr{A} \subset \mathscr{H}$.

Proof. We note that Lemmas 8 and 11 imply that $(\mathscr{H}, S(t))$ is a dissipative dynamical system which is asymptotically smooth. By Theorem 6 , it has compact global attractor $\mathscr{A}$ in the phase space $\mathscr{H}$.

\section{Conflicts of Interest}

The authors declare that they have no conflicts of interest.

\section{Acknowledgments}

This work is partly supported by the NSFC (nos. 11561064 and 11361053) and NWNU-LKQN-14-6.

\section{References}

[1] S. Woinowsky-Krieger, "The effect of axial force on the vibration of hinged bars," Journal of Applied Mechanics, vol. 17, pp. 35-36, 1950.

[2] H. M. Berger, "A new approach to the analysis of large deflections of plates," Journal of Applied Mechanics, vol. 22, pp. 465472, 1955. 
[3] J. E. Rivera and L. H. Fatori, "Smoothing effect and propagations of singularities for viscoelastic plates," Journal of Mathematical Analysis and Applications, vol. 206, no. 2, pp. 397-427, 1997.

[4] M. M. Cavalcanti, V. N. Domingos Cavalcanti, and T. Ma, "Exponential decay of the viscoelastic Euler-Bernoulli equation with a nonlocal dissipation in general domains," Differential and Integral Equations, vol. 17, no. 5-6, pp. 495-510, 2004.

[5] M. Conti and P. G. Geredeli, "Existence of smooth global attractors for nonlinear viscoelastic equations with memory," Journal of Evolution Equations, vol. 15, no. 3, pp. 533-558, 2015.

[6] C. M. Dafermos, "Asymptotic stability in viscoelasticity," Archive for Rational Mechanics and Analysis, vol. 37, no. 4, pp. 297-308, 1970.

[7] H. Xiao, "Asymptotic dynamics of plate equations with a critical exponent on unbounded domain," Nonlinear Analysis, Theory, Methods and Applications, vol. 70, no. 3, pp. 1288-1301, 2009.

[8] L. Yang and C.-K. Zhong, "Global attractor for plate equation with nonlinear damping," Nonlinear Analysis. Theory, Methods \& Applications, vol. 69, no. 11, pp. 3802-3810, 2008.

[9] G. Yue and C. Zhong, "Global attractors for plate equations with critical exponent in locally uniform spaces," Nonlinear Analysis. Theory, Methods \& Applications, vol. 71, no. 9, pp. 4105-4114, 2009.

[10] L. Yang, "Uniform attractor for non-autonomous plate equation with a localized damping and a critical nonlinearity," Journal of Mathematical Analysis and Applications, vol. 338, no. 2, pp. 1243-1254, 2008.

[11] A. Kh. Khanmamedov, "Existence of a global attractor for the plate equation with a critical exponent in an unbounded domain," Applied Mathematics Letters, vol. 18, no. 7, pp. 827-832, 2005.

[12] A. K. Khanmamedov, "Global attractors for the plate equation with a localized damping and a critical exponent in an unbounded domain," Journal of Differential Equations, vol. 225, no. 2, pp. 528-548, 2006.

[13] A. K. Khanmamedov, "A global attractor for the plate equation with displacement-dependent damping," Nonlinear Analysis. Theory, Methods \& Applications, vol. 74, no. 5, pp. 1607-1615, 2011.

[14] W. Ma and Q. Ma, "Attractors for stochastic strongly damped plate equations with additive noise," Electronic Journal of Differential Equations, no. 111, pp. 1-12, 2013.

[15] H. Wu, "Long-time behavior for a nonlinear plate equation with thermal memory," Journal of Mathematical Analysis and Applications, vol. 348, no. 2, pp. 650-670, 2008.

[16] A. R. A. Barbosa and T. F. Ma, "Long-time dynamics of an extensible plate equation with thermal memory," Journal of Mathematical Analysis and Applications, vol. 416, no. 1, pp. 143$165,2014$.

[17] M. A. Jorge Silva and T. F. Ma, "Long-time dynamics for a class of Kirchhoff models with memory," Journal of Mathematical Physics, vol. 54, no. 2, Article ID 021505, 2013.

[18] C. Giorgi, J. E. Rivera, and V. Pata, "Global attractors for a semilinear hyperbolic equation in viscoelasticity," Journal of Mathematical Analysis and Applications, vol. 260, no. 1, pp. 8399, 2001.

[19] C. Giorgi, V. Pata, and A. Marzocchi, "Asymptotic behavior of a semilinear problem in heat conduction with memory," Nonlinear Differential Equations and Applications, vol. 5, no. 3, pp. 333-354, 1998.
[20] C. Giorgi, M. Grasselli, and V. Pata, "Well-posedness and longtime behavior of the phase-field model with memory in a history space setting," Quarterly of Applied Mathematics, vol. 59, no. 4, pp. 701-736, 2001.

[21] X. Shen and Q. Ma, "The existence of random attractors for plate equations with memory and additive white noise," Korean Journal of Mathematics, vol. 24, no. 3, pp. 447-467, 2016.

[22] M. M. Cavalcanti, A. D. D. Cavalcanti, I. Lasiecka, and X. Wang, "Existence and sharp decay rate estimates for a von Karman system with long memory," Nonlinear Analysis: Real World Applications, vol. 22, pp. 289-306, 2015.

[23] I. Lasiecka and X. Wang, "Intrinsic decay rate estimates for semilinear abstract second order equations with memory," in New Prospects in Direct, Inverse and Control Problems for Evolution Equations, vol. 10 of Springer INdAM Series, pp. 271-303, Springer International Publishing, Cham, Switzerland, 2014.

[24] J. Zhou, "Global existence and blow-up of solutions for a Kirchhoff type plate equation with damping," Applied Mathematics and Computation, vol. 265, pp. 807-818, 2015.

[25] C. Giorgi, V. Pata, and E. Vuk, "On the extensible viscoelastic beam," Nonlinearity, vol. 21, no. 4, pp. 713-733, 2008.

[26] C. Giorgi, M. G. Naso, V. Pata, and M. Potomkin, "Global attractors for the extensible thermoelastic beam system," Journal of Differential Equations, vol. 246, no. 9, pp. 3496-3517, 2009.

[27] B. W. Feng, "Uniform attractors for a nonlinear nonautonomous extensible plate equation," Chinese Annals of Mathematics. Series A, vol. 37, no. 1, pp. 15-30, 2016.

[28] M. M. Cavalcanti, V. N. Domingos Cavalcanti, and J. A. Soriano, "Global existence and asymptotic stability for the nonlinear and generalized damped extensible plate equation," Communications in Contemporary Mathematics, vol. 6, no. 5, pp. 705-731, 2004.

[29] R. Temam, Infinite-Dimensional Dynamical Systems in Mechanics and Physics, vol. 68 of Applied Mathematical Sciences, Springer, New York, NY, USA, 1988.

[30] I. Chueshov and I. Lasiecka, Von Karman Evolution Equations: Well-Posedness and Long-Time Dynamics, Springer Monographs in Mathematics, Springer, New York, NY, USA, 2010.

[31] I. Chueshov and I. Lasiecka, "Long-time behavior of second order evolution equations with nonlinear damping," Memoirs of the American Mathematical Society, vol. 195, no. 912, 2008.

[32] I. Chueshov and I. Lasiecka, "Long-time dynamics of a semilinear wave equation with nonlinear interior/boundary damping and sources of critical exponents," in Control Methods in PDEDynamical Systems, vol. 426 of AMS Contemporary Mathematics, pp. 153-192, American Chemical Society, Providence, RI, USA, 2007. 


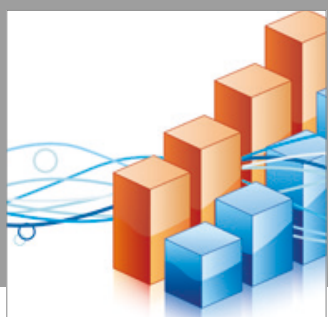

Advances in

Operations Research

vatersals

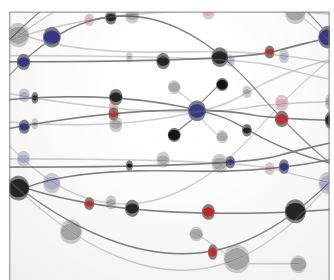

\section{The Scientific} World Journal
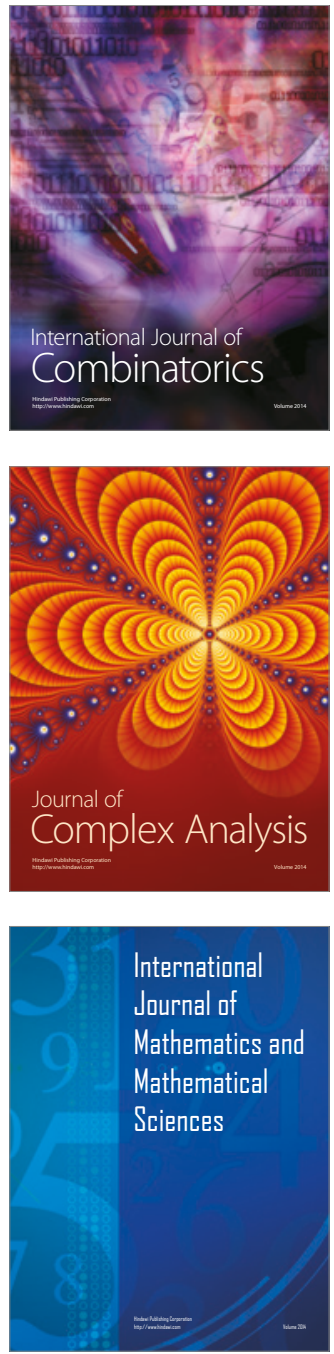
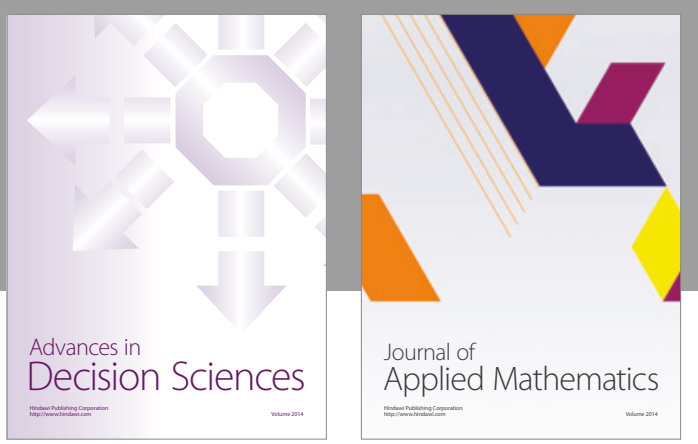

Algebra

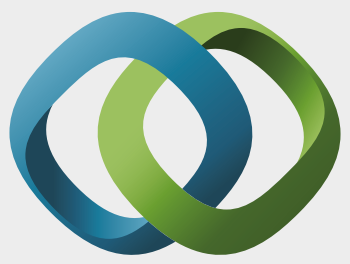

\section{Hindawi}

Submit your manuscripts at

https://www.hindawi.com
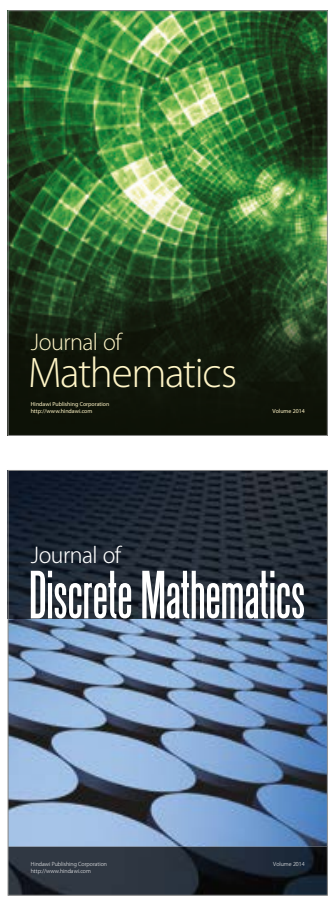

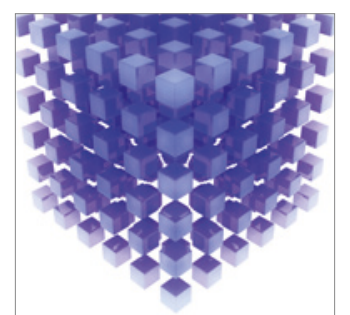

Mathematical Problems in Engineering
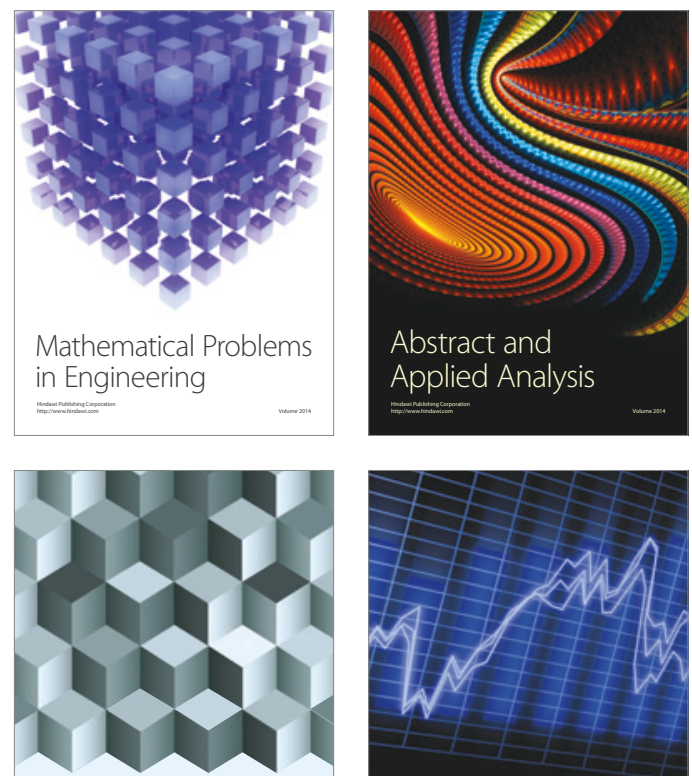

Journal of

Function Spaces

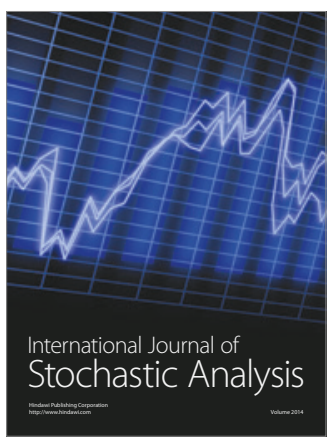

Probability and Statistics
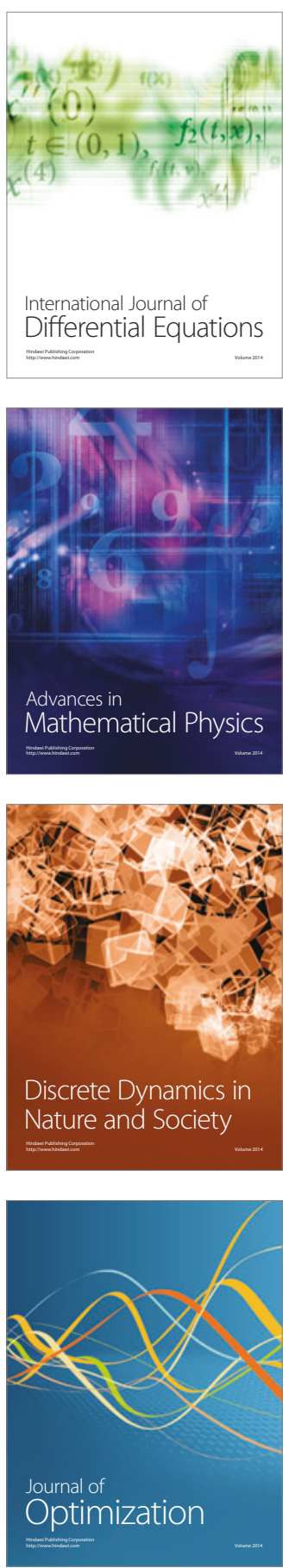